\title{
A Measurement Based Energy Model for IEEE 802.16e Mobile WiMAX Devices
}

\author{
Bjoern Dusza, Christoph Ide and Christian Wietfeld \\ Communication Networks Institute \\ TU Dortmund University \\ 44227 Dortmund, Germany \\ e-mail: \{Bjoern.Dusza, Christoph.Ide, Christian.Wietfeld\}@tu-dortmund.de
}

\begin{abstract}
The operational time of modern smart-phones with one filling of the accumulator is one of the most important performance parameter for the customers of new devices. This is the reason why extensive research has been performed in the fields of battery design and system optimization with regard on energy consumption. Due to the fact that the radio part of a smart-phone is one of the major energy consumers, a special focus has to be set on different approaches and strategies how to reduce the energy that has to be spent for the transmission of a certain amount of data. However, before new energy saving protocols can be efficiently developed one needs to have detailed knowledge on the different factors which impact the energy efficiency.

In this paper, we present a measurement based energy model for IEEE 802.16e conform Mobile WiMAX devices. For this purpose, extensive measurements of the energy consumption of a Mobile WiMAX USB Stick have been performed for different system parameterizations, different data sizes and different application data rates. From the results of the measurements analytic models have been derived which allow for the calculation of the energy that has to be spent per successfully submitted bit. These analytic formulas can now be integrated in system level simulators for the evaluation of the energy efficiency of newly designed protocols.
\end{abstract}

\section{INTRODUCTION}

The operational time for which a mobile phone can be used prior it has to be recharged is one of the most important performance parameter for the consumers of new smart-phones [1]. This leads to the major challenge that on the one hand new smart-phones with fast Central Processing Units (CPU), bright displays, lots of sensors and many different communication interfaces need more and more energy and on the other hand the battery technology is not developing as fast as it would be necessary. This is the reason why energy efficiency is one of the most important design targets if novel smart phones based on Long Term Evolution (LTE) or Mobile WiMAX have to be designed. However, before extensive simulations on the energy consumption of new systems, protocols or algorithms can be performed, detailed energy models are needed as a basis for the performance evaluation. One of the most critical performance indicators in this context is the energy that has to be spent for the successful submission of 1 bit.

In this paper, we present a detailed measurement based energy model for IEEE 802.16e Mobile WiMAX. The model describes the influence of the transmission power, the data size (e.g. in case of file transfer) and the application data rate (e.g. for streaming applications such as Voice over IP (VoIP))

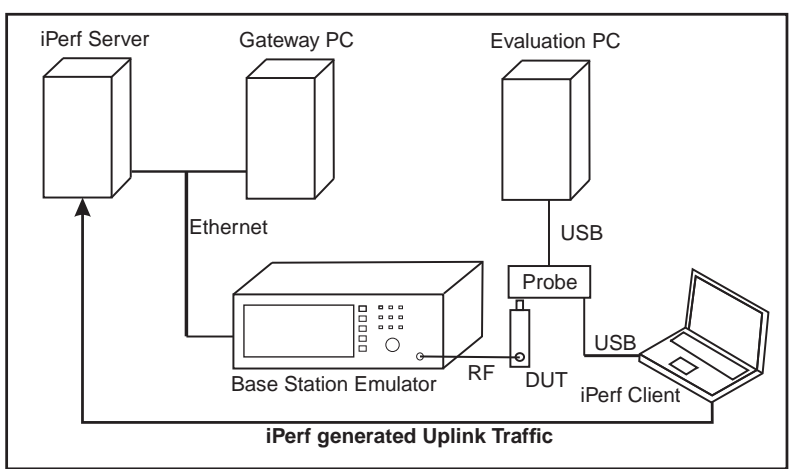

Fig. 1. Measurement Setup for Detailed Power Measurements

on the energy consumption per bit in closed formulas. For the derivation of the model extensive laboratory measurements have been performed based on a Mobile WiMAX base station emulator and a commercially available Universal Serial Bus (USB) enabled Device Under Test (DUT). For the measurement of the energy consumption, the current as well as the voltage at the input of the USB stick were sampled during an iPerf generated uplink data submission for different system parameterizations (see Fig. 1). The models show that all of the considered parameter have a major impact on the energy consumption per bit and therefore should be incorporated by future energy efficiency considerations. Exemplary concrete results presented in this paper are therefore the minimum amount of data that has to be collected for an energy efficient transmission as well as a proposal for optimized data aggregation schemes in wireless sensor networks.

\section{RElATED WORK}

Modeling the energy consumption of modern smart-phones is gaining importance during the last few years. This is due to the fact that cellular phones are no longer only phones but complete portable computer with lots of additional functions. This comes along with the fact that the power consumption is significantly increasing. In [2], an approach is presented which allows for the automated construction of energy models for Android based smart-phones. Instead of measuring the actual current drain from the battery, the method described here bases on the battery voltage sensor and knowledge about the battery discharge voltage curve. For the determination of 
TABLE I

MOBILE WiMAX SySTEM PARAMETRIZATION

\begin{tabular}{|c|c|}
\hline Parameter & Value \\
\hline Carrier Frequency [GHz] & $2.5405 \mathrm{GHz}$ \\
\hline Channel Bandwidth [MHz] & 10 \\
\hline UL Tx-power per Subcarrier & $-6 \mathrm{dBm}$ \\
\hline Fast Fourier Transform (FFT) Size & 1024 \\
\hline UL Modulation Scheme & QPSK \\
\hline UL Coding Rate & $1 / 2$ \\
\hline Coding Type & Convolutional Turbo Code (CTC) \\
\hline Duplexing Scheme & Time Division Duplex (TDD) \\
\hline Carrier Interleaving Scheme & Full Usage of Subchannels (FUSC) \\
\hline MIMO Type & 1 x 1 (SISO) \\
\hline Map Repetition Factors & 0 (No Repetition) \\
\hline HARQ & Disabled \\
\hline Maximum Transfer Unit (MTU) & 1368 Bytes \\
\hline
\end{tabular}

the energy consumption of different components of the device, the different parts are successively activated and deactivated by a training program which allows for the monitoring of the battery voltage change for each activation step. From that, power coefficients for a generic energy model are derived. Although the model covers the power consumption of the overall device, the radio parts for $\mathrm{WiFi}$ and $3 \mathrm{G}$ are only modeled by means of three states each (Idle, Forward Access Channel (FACH) and Dedicated Transport Channel (DCH) for 3G). A comparable approach can also be found in [3] where only two states (Idle and Active) for the energy consumption of the $3 \mathrm{G}$ radio interface are assumed.

A much more detailed investigation of the energy consumption of GSM, UMTS and WiFi has been performed in [4]. Here, the impact of different locations and different times are taken into consideration as well as user mobility. For the evaluation of the energy consumption the measured value is divided into a ramp-part, a data-part and a tail-part. The measurements show that for short data bursts the setup of the data channel as well as the termination causes significant costs in terms of energy consumption. Based on this knowledge a new protocol is proposed which optimizes the scheduling for a minimum energy consumption. It is worth noting that in [4] the assumption is used that the energy consumption is proportional to the length of transmission and the transmit power level. The results presented in this paper will show that this is not true under any circumstances for Mobile WiMAX.

Very detailed investigations on the energy consumption of WiFi systems have been performed in [5]. Here a measurement based approach was used to map the current power consumption to corresponding protocol events. Beside this, energy efficiency analyses have been performed in terms of energy per submitted bit.

\section{Measurement Setup}

The measurement setup used for the determination of the USB sticks energy consumption can be seen in Fig. 1. The actual bidirectional Mobile WiMAX link between the Base Station Emulator (BSE) and the DUT is realized by means of an RF cable to avoid external influences on the radio link.

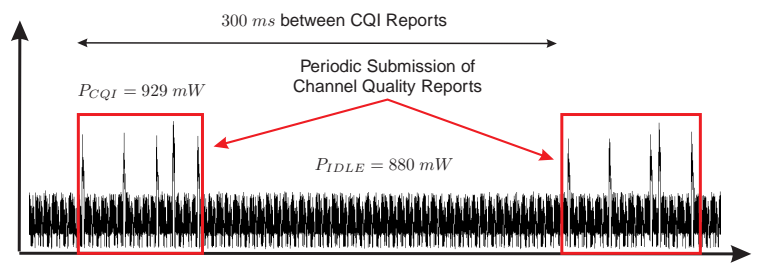

(a) Idle State with Channel Quality Transmission

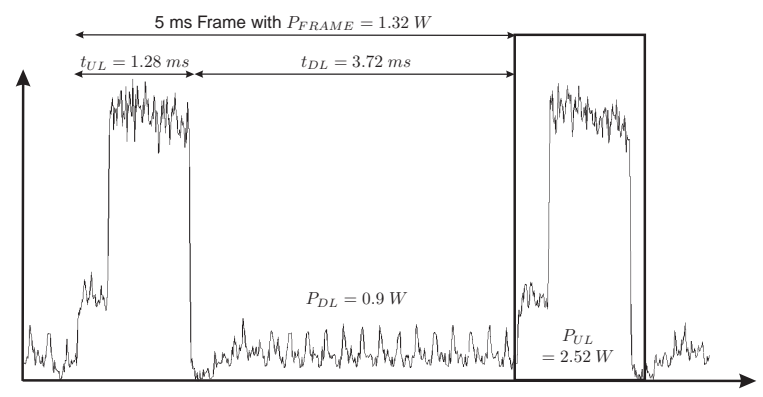

(b) Power Distribution for WiMAX Frame (DL/UL = 35/12)

Fig. 2. Mobile WiMAX Power Consumption Measurement

The BSE as the central component of the setup acts as a base station and therefore creates a standard conform radio cell towards the DUT. In the context of energy consumption measurements the main role of the BSE is the forwarding of the transmitted uplink data to the server as well as the submission of Medium Access Control (MAC) messages related to power control in the downlink. These messages are needed due to the fact that for the measurements a pure closed loop power control is applied which means that the uplink transmit power is solely set by the base station. This allows for full control of the measurements. The actual parametrization of the Mobile WiMAX link can be seen in Tab. I. The iPerf server, which acts as counterpart for the client PC, is connected to the BSE via Ethernet. Beside this, the BSE used for the investigations makes use of an additional gateway $\mathrm{PC}$ for end-to-end application testing. Some of the MAC mechanisms needed for this kind of test are executed on this external PC which is also connected to the BSE via Ethernet. The actual power measurement is performed by a measurement probe that is placed between the DUT and the client PC. Therefore, the energy consumed by the DUT has to pass the probe where it is measured in terms of electric current and voltage. From this, the current power consumption is calculated at a frequency of $100 \mathrm{kHz}$ and transmitted to an evaluation PC via USB. Here the data is stored for a detailed evaluation such as for example the determination of the consumed energy in a certain period of time. From this, it is for example possible to very precisely measure the energy that is consumed for the transmission of single symbols, bursts and frames as it can be seen in Fig. 2.

\section{RESUlts}

In this section the results of the energy consumption measurements are described in detail. A general overview on 
the course of the actual power over time can be seen from Fig. 2. Fig. 2(a) illustrates the power consumption over time for a Mobile WiMAX device that is connected to a base station but does not transmit or receive any data. Therefore a continuous average power consumption of $P_{I D L E}=880 \mathrm{~mW}$ is needed while every $300 \mathrm{~ms}$ a channel quality (CQI) report is submitted in the uplink. For this submission period the average power increases to $P_{C Q I}=929 \mathrm{~mW}$. Fig. 2(b) shows a zoom to the power measurements for one Time Division Duplex (TDD) frame. One can clearly see the different parts of the burst for the uplink and the downlink where the time ratio $t_{D L} / t_{U L}$ corresponds to the defined TDD downlink to uplink ratio of $35 / 12$. While the overall average power for the submission of one burst is $P_{F R A M E}=1.32 \mathrm{~W}$ only $P_{D L}=0.9 \mathrm{~W}$ are consumed during the reception phase while $P_{U L}=2.52 \mathrm{~W}$ are needed for the submission of data in the uplink (assuming an uplink Tx-power of $-6 \mathrm{dBm}$ per OFDM subcarrier as reported to the BS and Full Usage of Subchannels (FUSC)).

\section{A. Impact of the Transmit Power on the Energy Efficiency}

The influence of the uplink Tx-power per OFDM subcarrier (SC) on the energy needed for the successful submission of one bit was determined by submitting an iPerf generated data stream at the maximum possible data rate $D R_{\operatorname{Max}}$ for the different Downlink (DL) to Uplink (UL) ratios $\Psi$. During the transmission, the average power consumption was measured by using the setup described in section III. Afterwards the consumed energy $E$ for the submission of one bit was calculated by

$$
E[J / B i t]=\frac{\frac{1}{T} \cdot \int_{0}^{T} P(t) d t}{\frac{1}{T} \cdot \int_{0}^{T} D R_{M a x}(t) d t}
$$

for a defined measurement period $T$ of 30 seconds where $P$ denotes the actually measured power and $D R_{M a x}$ represents the maximum available data rate for a given system parametrization. For the concrete measurement assuming $10 \mathrm{MHz}$ channel bandwidth and FUSC the values for the transmission power per subcarrier can be converted to the overall transmission power by adding $27.5 d B$ (560 used

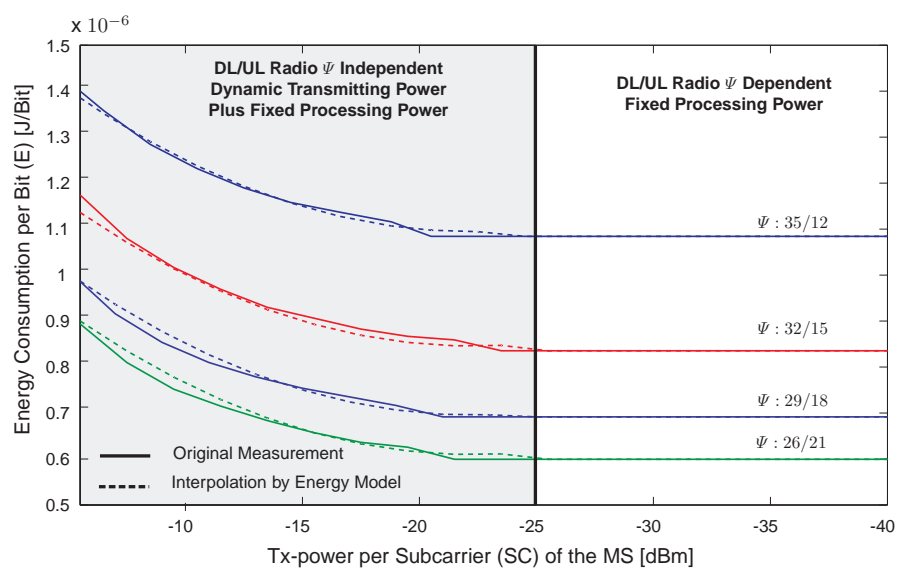

Fig. 3. Original Measurement vs. Analytical Energy Model data subcarrier). The results of the measurements can be seen from the solid lines in Fig. 3. The determined figures show that the overall power consumption can be divided in a fixed processing power that does only depend on the DL to UL ratio $\Psi$ and a Tx-power dependent component that is independent of $\Psi$ and added to the processing power. Polynomial fitting has been performed in a least square sense to find a polynomial that models the non-linear increase of the Tx-power dependent component. From that, we found that a second order polynomial is a very good approximation and the overall transmit power dependent energy consumption per bit can by analytically described as

$$
E\left(P_{\mathrm{Tx}}\right)= \begin{cases}C(\Psi) & , P_{\mathrm{Tx}} \leq P_{\mathrm{Tx}, M i n} \\ \alpha P_{\mathrm{Tx}}^{2}+\beta P_{\mathrm{Tx}}+\gamma+C(\Psi) & , P_{\mathrm{Tx}}>P_{\mathrm{Tx}, M i n}\end{cases}
$$

with the constants as shown in Tab. II. The results show that decreasing the Tx-power allows for massive energy savings. Therefore, the aim of protocol designers should be to spent only as much energy as necessary to meet the actual applications QoS requirements. A study on the relationship between the Signal to Noise and Interference Ratio (SNIR) and different QoS targets can be found in [6]. Nevertheless, under extremely good channel conditions it does not make sense to transmit at a Tx-power below $-25 \mathrm{dBm}$ per subcarrier. This is due to the fact that for very low Tx-power values the processing power is dominating the overall energy consumption. In this case, the transmit power of $-25 \mathrm{dBm}$ per subcarrier should, from an energy efficiency point of view, be used to ensure an optimum signal quality even if lower power would be sufficient for a successful transmission. Nevertheless, interference avoidance considerations could lead to a further decreases of the Tx-power.

\section{B. Impact of the App. Data Rate on the Energy Efficiency}

Due to the fact that typical applications of broadband wireless networks include real-time applications such as VoIP or video streaming the energy efficiency in terms of energy consumed for the successful submission of one bit has been determined for different application data rates. From an energy efficiency perspective the continuous submission of data with a data rate below the maximum is something quite different than

TABLE II

Parameter Set For TX-POWER DePEndent ENERgy EFficiency

\begin{tabular}{|c|c|}
\hline Parameter & Value \\
\hline$\alpha$ & $1.0325 \cdot 10^{-6}$ \\
\hline$\beta$ & $4.6000 \cdot 10^{-5}$ \\
\hline$\gamma$ & $5.2250 \cdot 10^{-4}$ \\
\hline$C(\Psi=35 / 12)$ & $1.1084 \cdot 10^{-3}$ \\
\hline$C(\Psi=32 / 15)$ & $8.3530 \cdot 10^{-4}$ \\
\hline$C(\Psi=29 / 18)$ & $6.9160 \cdot 10^{-4}$ \\
\hline$C(\Psi=26 / 21)$ & $5.9940 \cdot 10^{-4}$ \\
\hline$P_{\mathrm{Tx}, \text { Min }}$ & $-25 d B m / S C$ \\
\hline
\end{tabular}


the submission of one big file (e.g. file transfer). Therefore, measurements comparable to those described in the previous section have been performed for variable application data rates and a fixed uplink Tx-power of $-6 \mathrm{dBm} / S C$. The results of the investigations can be seen in Fig. 4. The linear shape of the energy per bit figure in a double logarithmic plot and the negative slope lead to a $1 / x$ relationship between the application data rate and the energy efficiency. We found that the energy consumed for one bit can be analytically modeled as

$$
E\left(D R_{A p p}\right)=\frac{9.7 \cdot 10^{-4}}{D R_{A p p}^{0.94}}
$$

where $D R_{A p p}$ denotes the application data rate. Although one can see from Fig. 4 that the impact of the DL to UL ratio $\Psi$ is negligible for different application data rates, the zoom to the maximum data rate area shows an interesting detail: For application data rates below the $\Psi$ dependent maximum (e.g. in case of VoIP) the best choice for reducing the energy consumption is to apply a DL/UL ratio with less UL resources. Nevertheless the highest overall efficiency can be achieved by means of $\Psi=26 / 21$, but only if the maximum available data rate is occupied by the application.

From the results one can conclude that the maximum energy efficiency can be achieved in case of a maximum utilization of the link. For the example of wireless sensor networks this would mean that not every node should be maintaining a Mobile WiMAX link but only one aggregation node which collects the data via an energy efficient local network such as Zigbee. This aggregation node would than be able to optimally utilize the link and therefore minimize the overall energy consumption.

\section{Impact of the File Size on the Energy Efficiency}

For the determination of the energy efficiency in case of an uplink file transfer, files with different sizes have been

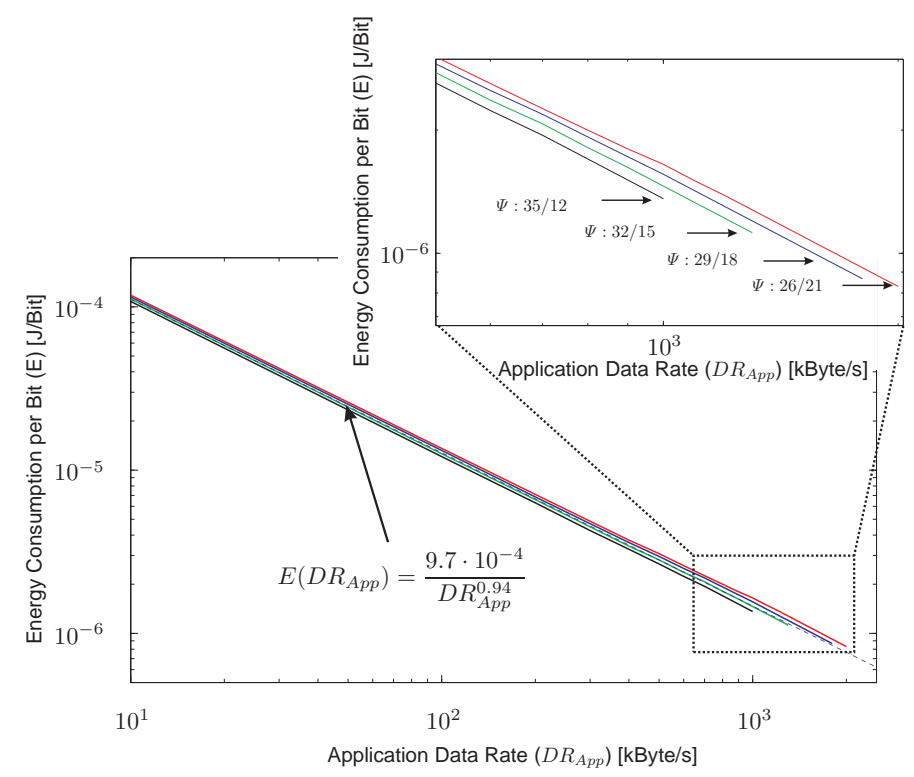

Fig. 4. Energy Model for Different Application Data Rates

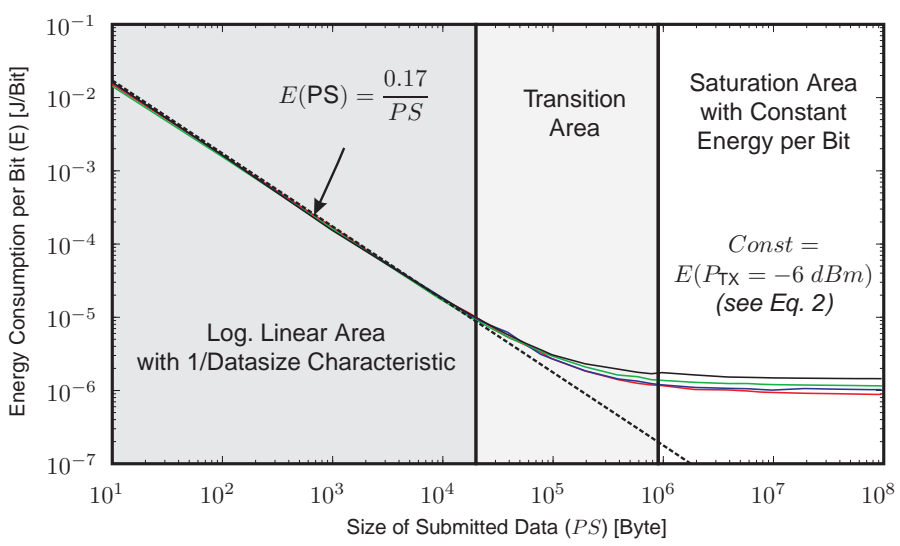

Fig. 5. Energy Efficiency for Different Amounts of Data @-6 dBm Tx-power

transfered. In contrast to the previously described models for this investigation the energy consumption per bit was calculated as

$$
E[J / B i t]=\frac{\int_{0}^{T_{P S}} P(t) d t}{P S}
$$

where $T_{P S}$ determines the time that is needed to submit a file of $P S$ bits. The results plotted in Fig. 5 show that the energy consumption per bit has to be modeled for three different areas. For a packet size of up to $20 k$ Byte the shape is linear in a double logarithmic plot which again leads to a $1 / x$ relationship. For packet sizes above $900 k$ Byte the energy consumption per bit is constant and can be calculated based on the UL/DL ratio $\Psi$ and the Tx-power applying equation (2). For the transition area between $20 k$ Byte and $900 k$ Byte either the analytical expression for lower packet sizes or higher packet sizes can be applied but increased errors have to be expected. The overall energy model for the packet size $P S$ dependent energy consumption can be analytically expresses as:

$$
E(P S)= \begin{cases}\frac{0.17}{P S} & P S<20 k B y t e \\ \text { Const } & P S>900 k B y t e\end{cases}
$$

From the results one can conclude that from an energy efficiency perspective the optimal Mobile WiMAX packet scheduler is one that waits until at least $900 \mathrm{kByte}$ of data are awaiting submission before it requests the necessary bandwidth from the base station.

\section{Generality for Different Chip-Sets and Frequencies}

Due to the fact that all of the previously described measurements have been performed based on the same device, an additional validation measurement has been performed using a Mobile WiMAX USB stick from an other manufacturer which is using a different chip-set. Furthermore, the stick applied for the validation works at a center frequency of $3.5 \mathrm{GHz}$ instead of $2.5 \mathrm{GHz}$. Fig. 6 shows the plot of the energy efficiency as a function of the data size as an exemplary measurement using this second device. One can see from the comparison with Fig. 5, that the overall behavior of the device is comparable to the $2.5 \mathrm{GHz}$ device especially for 


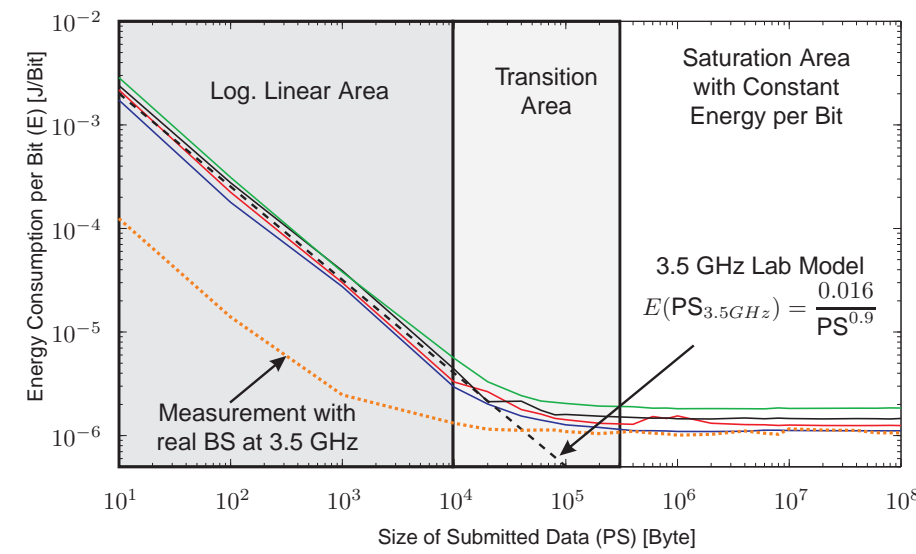

Fig. 6. Model Validation Measurement with different Devices in the Lab and Outdoor

large packet sizes. Beside this, the dimensions of the different areas are comparable for both devices. Nevertheless, there are also differences between the energy efficiencies of the two different DUTs. The most important one is the significantly decreased energy consumption per bit of the $3.5 \mathrm{GHz}$ stick for small data sizes. The reason for this behavior is a tail energy that is consumed by the $2.5 \mathrm{GHz}$ device after the termination of the actual data transfer. This behavior is not observable for the $3.5 \mathrm{GHz}$ device which leads to a decreased energy consumption especially for small data sizes. Finally, an additional measurement has been performed using a real base station instead of the emulator (see Fig. 7). The results are shown in Fig. 6 (dotted line). One can see, that for larger packet sizes the energy efficiency equals the efficiency of the other sticks but for small packets the stick used for the real world measurements is significantly more efficient than the other devices. One possible explanation would be, that the device used for the real world validation is the newest revision of the stick used for the $3.5 \mathrm{GHz}$ lab test and proceedings in terms of energy efficiency have been made. Beside this, it has to be noted that for the real world measurement no control of the system parameters such as Tx-power and DL/UL ratio have been possible. Therefore, it is hard to explain the reasons for the differences in detail. Nevertheless, one can summarize that the derived energy models in general are valid for different USB based Mobile WiMAX devices although device specific offsets may have to be applied for different chip-sets and frequency bands.

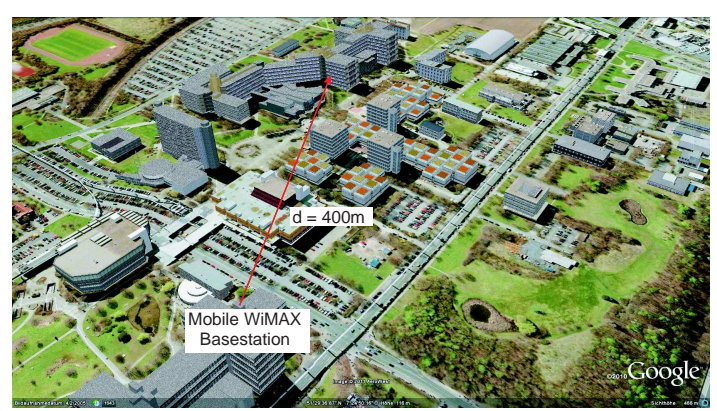

Fig. 7. Mobile WiMAX Link for Validation Measurement, (c) 2011 Google

\section{CONCLUSIONS}

In this paper we have presented a detailed energy model for IEEE 802.16e conform Mobile WiMAX devices. Therefore, extensive measurements of the energy consumption per bit have been performed for different uplink Tx-power, different application data rates and different packet sizes. From all of the measurements closed analytical expressions have been derived which allow for the application of the models in for example system level simulators. From the results the following direct conclusions can be derived for the design of an energy efficient Mobile WiMAX based network:

- The transmit power should be as low as possible. Nevertheless it should, from an energy efficiency perspective, not be below $-25 \mathrm{dBm}$ per subcarrier because for very low transmission power no further energy savings are possible.

- In wireless sensor networks as few nodes as possible should maintain a Mobile WiMAX connection due to their low application data rate. Instead of this, aggregation nodes should be used which collect the data via for example Zigbee. Applying this architecture allows for a higher utilization of the Mobile WiMAX link and therefore an optimized energy efficiency.

- If non real time data has to be transmitted it is useful to collect at least $900 \mathrm{kByte}$ of data before uplink resources are requested by the scheduler. For lower packet sizes the energy efficiency is significantly decreasing.

\section{ACKNOWLEDGMENT}

Part of the work on this paper has been supported by Deutsche Forschungsgemeinschaft (DFG) within the Collaborative Research Center SFB 876 "Providing Information by Resource-Constrained Analysis", projects A4 and B4.

\section{REFERENCES}

[1] L. Bloom, R. Eardley, E. Geelhoed, M. Manahan, and P. Ranganathan, Investigating the Relationship Between Battery Life and User Acceptance of Dynamic, Energy-Aware Interfaces on Handhelds, in Proc. Mobile HCI, 2004, pp.13-24.

[2] L. Zhang, B. Tiwana, Z. Qian, Z. Wang, R. Dick, Z. Mao and L. Yang, Accurate Online Power Estimation and Automatic Battery Behavior Based Power Model Generation for Smartphones, in Proc. of the 8th IEEE/ACM/IFIP International Conference on Hardware/Software Codesign and System Synthesis, p. 105-114, ACM, Scottsdale, USA, 2010

[3] M. B. Kjaergaard, J. Langdal, T. Godsk, and T. Toftkjaer, EnTracked: Energy-Efficient Robust Position Tracking for Mobile Devices, in Proc. of the 7th international conference on Mobile systems, applications and services, p. 221-234, ACM, Krakow, Poland, 2009

[4] N. Balasubramanian, A. Balasubramanian, and A. Venkataramani, Energy Consumption in Mobile Phones: A Measurement Study and Implications for Network Applications, in Proc. of the 9th ACM SIGCOMM conference on Internet measurement, p. 280-293, ACM, Chicago, USA, 2009

[5] A. Rice and S. Hay, Decomposing Power Measurements for Mobile devices, in Prof. of the IEEE International Conference on Pervasive Computing and Communications (PerCom), p. 70 -78, Mannheim, Germany, 2010

[6] C. Ide, B. Dusza, C. Wietfeld, Mobile WiMAX Performance Measurements with Focus on Different QoS Targets, in Proc. of the 18th IEEE International Workshop on Local and Metropolitan Area Networks (LANMAN), Chapel Hill, USA, 2011 